\title{
Fertilité de brebis Pelibuey et Suffolk en climat tropical
}

\author{
J.L. Fuentes ${ }^{1}$ et P. Chemineau 2* \\ 1 CIMA, Carretera Central, km 21,5 Cotorro, Ciudad de La Habana, Cuba; \\ 2 INRA, Station de physiologie de la reproduction, Nouzilly, 37380 Monnaie, France
}

(reçu le 28 octobre 1988, accepté le 27 février 1989)

\begin{abstract}
Résumé - Cinquante brebis Pelibuey (race locale) et 47 brebis Suffolk (importées depuis 3 ans d'Amérique du Nord) sont utilisées dans une expérience factorielle $2 \times 2$ (race femelle $\times$ race mâle), réalisée dans l'île de Cuba (latitude $22^{\circ}$ Nord, température maximum de l'air $31^{\circ} 8 \mathrm{C}$ ). La moitié des femelles de chaque race sont inséminées, sur œstrus naturel, avec des mâles de l'autre race, l'autre moitié avec des mâles de la même race. Le rythme respiratoire et la température rectale de chaque brebis ont été mesurés le jour de l'insémination, 8-10 et 17-18 jours plus tard, afin d'apprécier leur adaptation aux conditions du climat tropical.

Quel que soit le mâle d'insémination, la fertilité des femelles Suffolk est toujours beaucoup plus faible que celle des femelles Pelibuey ( $25 \%$ contre $74 \%$, respectivement; $P<0,01)$. En revanche, quelle que soit la race de la femelle, la fertilité n'est pas différente, que les béliers soient Suffolk ou Pelibuey ( $44 \%$ contre $57 \%$, respectivement). Aux trois moments de mesure le rythme respiratoire des brebis Suffolk est environ deux fois plus élevé que celui des femelles Pelibuey (105 contre 45 resp./min, respectivement; $P<0,001)$ et leur température rectale est supérieure de $0,9{ }^{\circ} \mathrm{C}(39,8$ contre $38,9^{\circ} \mathrm{C}$, respectivement; $P<0,001$ ).
\end{abstract}

brebis - race - fertilité - température - tropiques

Summary - Fertility of Pelibuey and Suffolk ewes in a tropical climate. Fifty Pelibuey ewes (local breed) and 47 Suffolk ewes (imported as of 3 years ago from North America) were used in a factorial $2 \times 2$ (ewe breed $\times$ ram breed), carried out on the island of Cuba $\left(22^{\circ}\right.$ North; maximum air temperature $31.8^{\circ} \mathrm{C}$ ). Half of the females, in each breed, were inseminated on natural cestrus, with males from the other breed, the other half being inseminated with rams from the same breed. Respiratory rhythm and rectal temperature of each ewe were measured on the day of insemination, 8-10 and 17-18 days later, in order to appreciate their adaptation to a tropical climate.

Whatever the breed of ram used for insemination, the conception rate of Suffolk ewes was significantly lower than that of Pelibuey ewes $(25 \%$ vs $74 \%$, respectively; $\mathrm{P}<0.01 ;$ Table 1$)$. On the contrary, whatever the ewe breed, the fertility was not significantly different between Pelibuey and Suffolk rams (44\% vs $57 \%$, respectively). At the three times of measurements the respiratory rhythm of Suffolk ewes was about twice that of Pelibuey ewes (105 vs 45 resp./min, respectively; $\mathrm{P}<0.001)$ and their rectal temperature was $0.9{ }^{\circ} \mathrm{C}$ higher $\left(39.8^{\circ}\right.$ vs $38.9^{\circ} \mathrm{C}$, respectively; $\mathrm{P}<0.001$; Table (1). Within breed, a significant effect of time of measurement was observed on both parameters; respiratory rate and rectal temperature were higher on the day of insemination than the two other days of measurement.

It was concluded that Suffolk ewes, in a tropical area, had a lower fertility than the local Pelibuey breed.

ewe - breed - fertility - temperature - tropics

* Tirés à part : P. Chemineau, à l'adresse ci-dessus. 


\section{INTRODUCTION}

L'importation d'animaux sélectionnés, en provenance des żones tempérées, est une voie intéressante pour l'amélioration rapide de certains caractères de production qui font défaut aux animaux des zones tropicales. II convient cependant de prendre garde aux difficultés d'adaptation de certaines races aux contraintes de l'environnement tropical. Dans les conditions de Cuba les brebis Suffolk ont des difficultés de reproduction, puisque plus de la moitié des femelles des troupeaux d'élevage de cette race, ne mettent pas bas tous les ans.

Nous avons voulu savoir dans l'expérience décrite ici, quelle était, dans ces conditions climatiques, la fertilité réelle des brebis Suffolk (génération importée d'un pays d'Amérique du Nord, depuis trois ans), comparée à celle des Pelibuey (race locale de Cuba; Fuentes et al., 1983) et quelle était la part, dans nos conditions locales de milieu, du mâle et de la femelle dans la mauvaise fertilité attendue de la race importée.

\section{MATÉRIEL ET MÉTHODES}

\section{Milieu physique et conditions d'éle- vage}

L'expérience est réalisée en octobre-novembre dans l'île de Cuba (latitude $22^{\circ}$ Nord). C'est la fin de la saison chaude, la température minimale est, en moyenne, de $19,5^{\circ} \mathrm{C}$ et la température maximale de $31,8^{\circ} \mathrm{C}$. L'hygrométrie relative quotidienne varie entre $80 \%$ et $84 \%$. La température ambiante, à proximité immédiate des animaux, au moment des mesures $(14 \mathrm{~h})$ a varié de $22^{\circ} \mathrm{C}$ à $29,5^{\circ} \mathrm{C}$. Tous les animaux sont maintenus en stabulation (à l'ombre) pendant toute la durée de la période expérimentale; ils reçoivent une alimentation à base de Merker, distribué ad libitum et d'aliment concentré. Ils disposent d'eau en permanence, à volonté.

\section{Animaux et plan expérimental}

Cinquante brebis Pelibuey adultes et 47 brebis Suffolk sont utilisées. Les brebis Pelibuey ont toutes déjà mis bas au moins une fois alors que les brebis Suffolk, présentes à Cuba depuis 3 ans, n'ont jamais mis bas.

Le plan expérimental adopté est un schéma factoriel $2 \times 2$ (race mâle $\times$ race femelle) :

- les 50 femelles Pelibuey sont inséminées avec des mâles Pelibuey ( 25 femelles) ou Suffolk ( 25 femelles);

- les 47 femelles Suffolk sont inséminées avec des mâles Pelibuey (24 femelles) ou Suffolk (23 femelles).

Les mêmes mâles sont utilisés d'un groupe de femelles à l'autre.

\section{Détections d'œestrus et inséminations}

L'expérience est réalisée entièrement sur cestrus naturels, qui sont détectés tous les matins du 15 octobre jusqu'au 9 novembre. Pendant cette période, les femelles sont réparties dans les différents lots expérimentaux. Elles sont inséminées par saillie "en main" (= SN, la moitié des brebis de chaque lot), ou par insémination artificielle avec du sperme frais (= IA, l'autre moitié des brebis de chaque lot). Les saillies et/ou l'insémination artificielle sont réalisées une seule fois dans les $5 \mathrm{~h}$ qui suivent la détection de l'œstrus.

\section{Mesures et enregistrements}

Le jour de l'insémination, 8 à 10 jours et 17 à 18 jours après l'insémination, le rythme respiratoire et la température rectale sont enregistrés, sur chaque femelle, aux heures les plus chaudes de la journée (14 h). 
Un diagnostic précoce de gestation est réalisé 17 ou 18 jours après l'insémination par dosage de la progestérone plasmatique (Kit de I'International Atomic Energy Agency, Vienne, Autriche). Les premiers retours en œstrus sont détectés, la date et le nombre d'agneaux sont enregistrés à la mise bas.

\section{Analyse des résultats}

Les comparaisons entre pourcentages ont été réalisées avec un test du $\chi^{2}$ ou un test des probabilités vraies. Les comparaisons entre moyennes par analyse de variance de mesures répétées puis par un test $t$, apparié ou non (Dagnélie, 1970).

\section{RÉSULTATS}

La fertilité ne varie pas significativement avec le mode d'insémination ( $S N: 25 / 49$ contre IA : 24/48); en conséquence, les résultats des deux types d'insémination ont été confondus.
Quelle que soit la race de la femelle, la fertilité n'est pas significativement différente d'une race de mâle à l'autre (Pelibuey : $57 \%$ contre Suffolk : $44 \%$; Tableau 1). En revanche, quelle que soit la race du mâle d'insémination, les femelles Pelibuey ont une fertilité très significativement supérieure à celle des femelles Suffolk $(74 \%$ contre $25 \%$ respectivement, Tableau I).

Toutes les femelles diagnostiquées gestantes à 17-18 jours mettent bas sauf une brebis Suffolk; une brebis Pelibuey diagnostiquée vide, met bas. Toutes les femelles diagnostiquées vides, sauf cette dernière brebis, reviennent en œstrus au bout d'un cycle.

La prolificité n'est pas significativement différente d'une race de femelles à l'autre (Suffolk : 1,5 agneau/mise bas contre Pelibuey: 1,4).

Les effets de la race, du moment de mesure et de l'interaction race $x$ moment de mesure sont très significatifs sur le rythme respiratoire $(P<0,001)$. A chacun des moments de mesure, le rythme respiratoire des brebis Suffolk est toujours très significativement supérieur à celui des

Tableau I. Fertilité, en \% de femelles mettant bas, de brebis Pelibuey et Suffolk accouplées avec des mâles Pelibuey ou Suffolk. CEstrus naturel; le nombre de brebis figure entre parenthèses.

\begin{tabular}{llll}
\hline Race de la femelle & \multicolumn{2}{c}{ Race du mâle } & $\begin{array}{l}\text { Différence } \\
\text { statistique } \\
\text { (race mâle) }\end{array}$ \\
& Pelibuey & Suffolk & NS \\
\hline $\begin{array}{l}\text { Pelibuey } \\
\text { Suffolk }\end{array}$ & $84(25)$ & $64(25) / 74(50)$ & NS \\
& $29(24)$ & $22(23) / 25(47)$ & NS \\
Différence statistique & $57(49)$ & $44(48)$ & \\
(race femelle) & $P<0,001$ & $P<0,01 \quad P<0,001$ & \\
\hline
\end{tabular}


brebis Pelibuey : près du double le jour de l'insémination et plus du double les deux autres jours de mesure (Tableau II). Le rythme respiratoire des brebis Pelibuey diminue entre les mesures 1 et 2 ( $P$ $<0,001)$ et entre les mesures 2 et $3(P<$ 0,01 ), celui des brebis Suffolk baisse entre les mesures 1 et $2(P<0,05)$ mais pas entre les mesures 2 et 3 .

Les effets de la race et du moment de mesure, mais pas de l'interaction, sont très significatifs sur la température rectale $(P<0,001)$. La température rectale des brebis Suffolk est également toujours très significativement supérieure à celle des brebis Pelibuey : $+0,8^{\circ} \mathrm{C}$ d'écart le jour de l'insémination et $+0,9^{\circ} \mathrm{C}$ d'écart les deux autres jours. La température rectale des deux groupes de brebis est significativement plus élevée le jour de l'insémination que les deux autres jours (Pelibuey: $P<0,001$; Suffolk : $P<0,05$; Tableau II).

\section{DISCUSSION}

La comparaison des résultats de fertilité entre les deux races de brebis montre que les brebis Pelibuey ont des performances très supérieures à celles des femelles Suffolk, dans ces conditions expérimentales. Malgré leur venue en chaleurs la plupart des brebis Suffolk ne sont pas gestantes au bout d'un cycle et reviennent en œstrus, sans que la durée du cycle soit modifiée de façon notable. En effet, la fertilité des deux lots de brebis Suffolk, que celles-ci soient accouplées avec un mâle Suffolk ou avec un mâle Pelibuey, est 3 fois plus faible que celle des deux lots de brebis Pelibuey. La race du mâle utilisé pour la saillie ne modifie pas significativement la fertilité des femelles, bien que celle-ci soit légèrement plus faible pour les béliers Suffolk que pour les béliers Pelibuey.

Tableau II. Rythme respiratoire et température rectale de brebis Pelibuey et Suffolk le jour de l'insémination (mesure $\left.N^{\circ} 1\right), 8-10$ jours $\left(N^{\circ} 2\right)$ et 17-18 jours $\left(N^{\circ} 3\right)$ après l'insémination. Moyenne \pm écart type.

$\begin{array}{lll}\text { Pelibuey } & \text { Suffolk } & \text { Effet Race: } \\ (\mathrm{N}=50) & (\mathrm{N}=47) & \text { R. resp. }\end{array}$

Temp. rect.

Mesure 1

$\begin{array}{lcccc}\text { R. resp. (resp/min) } & 61 \pm 19 & 110 \pm 27 & P<0,001 & P<0,001 \\ \text { Temp. rect. }\left({ }^{\circ} \mathrm{C}\right) & 39,1 \pm 0,4 & 39,9 \pm 0,5 & & \end{array}$

Mesure 2

$\begin{array}{lcccc}\text { R. resp. } & 40 \pm 12 & 100 \pm 24 & P<0,001 & P<0,001 \\ \text { Temp. rect. } & 38,8 \pm 0,4 & 39,7 \pm 0,4 & & \end{array}$

Mesure 3

$\begin{array}{lcccc}\text { R. resp. } & 34 \pm 8 & 107 \pm 23 & P<0,001 & P<0,001 \\ \text { Temp. rect. } & 38,8 \pm 0,4 & 39,7 \pm 0,5 & & \end{array}$

Effet moment de mesure

(races confondues)
R. resp.
$P<0,001$
Temp. Rect.
$P<0,001$ 
La cyclicité installée chez les femelles des deux races laisse penser que chez les brebis Suffolk, comme chez les brebis Pelibuey, les ovulations se sont produites normalement en même temps que les chaleurs. L'absence de gestation a probablement pour origine une mortalité embryonnaire très précoce, sans doute avant les jours 10-12 du développement de l'embryon où celui-ci envoie le "signal" qui évite la lutéolyse (Martal \& Charlier, 1985) et donc le retour en œstrus. Les températures élevées provoquent, en effet, lorsque les brebis sont en stress thermique, une mortalité embryonnaire très précoce, sans modifier notablement le cycle œstrien (Thwaites, 1967, 1969; Entwistle, 1973).

Les enregistrements réalisés concernant le rythme respiratoire et la température rectale montrent, en effet, que les brebis Suffolk ont peut-être dépassé le seuil d'inconfort thermique et qu'elles ont mis en route leurs mécanismes de thermorégulation (Herz \& Steinhauf, 1978; Berbigier, 1988). En effet, quel que soit le jour de mesure, les brebis Suffolk de cette expérience, ont des rythmes respiratoires et des températures rectals du même ordre que ceux des brebis placées en chambre chaude par Thwaites (1967), alors que ces mêmes paramètres, chez les brebis Pelibuey, sont comparables à ceux des brebis témoins, non stressées. Les brebis Pelibuey, en effet, ne manifestent pas de signe évident d'hyperthermie, malgré un rythme respiratoire et une température rectale plus élevés le jour de l'insémination. Cette réaction thermorégulatrice leur permet, très probablement, d'aboutir à de très bonnes performances de fertilité.

Les résultats présentés ici indiquent donc qu'il existe des différences très importantes dans les réactions physiologiques des races à supporter un stress thermique. La fonction de reproduction de la brebis Suffolk, race d'origine tempérée importée sous les tropiques, pourrait être affectée par la chaleur.

\section{REMERCIEMENTS}

Les auteurs souhaitent remercier les membres du personnel de la Estacion Ovina du CIMA pour leur aide ainsi que $D$. Chupin pour ses critiques constructives pendant l'expérimentation.

\section{RÉFÉRENCES}

Berbigier P. (1988) Bioclimatologie des Ruminants Domestiques en Zone Tropicale. INRA Paris

Dagnelie P. (1970) Théorie et Méthodes Statistiques. Vol. Il. Presses Agronomiques de Gembloux, Belgique, pp. 21-29, 98-105

Entwistle K.W. (1973) Ram fertility and fertilization rates in the ewe in a semi-arid tropical environment. Int. J. Biometeorol. 17, 109-113

Fuentes J.L., Lima T., Pulenets N., Pavon M., Albuernes R., Sans V. \& Peron N. (1984) Some aspects of the reproductive performance of the Pelibuey ewe in Cuba. In : Reproduction des Ruminants en Zone Tropicale. Pointe-à-Pitre (FWI), 8-10 juin 1983. INRA Publ. (Les Colloques de l'INRA, $n^{\circ} 20$ ), pp. 135-145

Herz A. \& Steinhauf D. (1978) The reaction of domestic mammals to heat stress. Animal Research and Development 7, 7-38

Martal J. \& Charlier M. (1985) Avortements précoces et signaux embryonnaires de reconnaissance de la gestation. Rec. Méd. Vét. 161, 8797

Thwaites C.J. (1967) Embryo mortality in the heat stressed ewe. I. The influence of breed. J. Reprod. Fertil. 14, 5-14

Thwaites C.J. (1969) Embryo mortality in the heat stressed ewe. II. Application of hot-room results to field conditions. J. Reprod. Fertil. 19, 225-262 